\title{
The design of a composite folding bike to improve the user experience of commuters
}

\author{
Thomas Vervisch ${ }^{1}$, Yannick Christiaens ${ }^{1}$ and Jan Detand ${ }^{1, a}$ \\ ${ }^{1}$ Ghent University, Faculty of Engineering and Architecture, Department of Industrial System and Product Design, \\ 8500 Kortrijk, Belgium
}

\begin{abstract}
Over the last years, the popularity of folding bikes has been increasing as a result of the rise of multi-modal transport. They are used by commuters as a complement to public transport. Despite the increasing popularity, the current market offer of folding bikes still represents quite some restrictions and downsides which decrease their usability. This paper shows a user-centred process of designing and prototyping a composite folding bike with the aim of improving the user experience of folding bike using commuters. By improving the usability and ease of use of folding bikes, their full potential can be unlocked. The design process led to a disruptive folding bike design with front and rear single-sided offset wheel mounting. The concept excels in its intuitive and quick folding mechanism, superior riding performance and comfort, adjustability and overall ease of use. In addition to the design process and prototyping of the folding bike, this extended abstract elaborates on the performed user tests and its results. These tests range from the impact of offset wheels to the intuitiveness of the folding mechanism and were performed in order to prove different aspects of the design. This research shows how rethinking and redesigning a bike concept (product) from scratch, using a user-centred design process and taking into account the three aspects - business, technology and people - can lead to a disruptive design that improves usability and the overall user experience of the stakeholders.
\end{abstract}

\section{Introduction}

Despite the recent increasing research and innovation in the world of folding bikes [1-2], current market offer does not meet the desires of commuters using them for multimodal commute. The need for a folding bike that is designed to fulfil the demands and wishes of present-day commuters initiated this design process. The project started with initial user tests in which test subjects had to execute each aspect of a folding bike commuter's customer journey. These tests revealed several shortcomings of the current market offer of folding bikes. The first main deficiency is the complexity of the folding mechanisms with safety locks. This leads to a lack of intuitiveness of the folding mechanisms. In addition, the need for skilled and precise handling leads to a bike that does not fold fast and easy enough for the rushed daily use while commuting. A second important drawback is that most folding bikes are chunky and too heavy, making them uncomfortable and a big burden to carry. This is because they are made out of traditional bike-building materials, such as aluminium and steel alloys.

\footnotetext{
${ }^{\text {a }}$ Corresponding author: Jan.Detand@UGent.be
} 
A third flaw is the abundance of exposed or poorly incorporated parts, such as exposed brake cables or externally mounted lights and reflectors. These elements obstruct the ease of use. A last main weakness of folding bikes is the small wheels. Folding bikes often have 20 " or even 16 " wheels. This results in a lack of comfort and even sketchy riding characteristics, especially at higher speeds.

This extended abstract explores solutions to these shortcomings and the user-centered design process of a concept folding bike, while taking into account the three design thinking aspects: business, technology and people. It furthermore elaborates on comparative user tests of the intuitiveness, the folding speed and the riding experience of the concept. These tests were performed in order to improve the usability of the folding bike and the user experience of the commuter, but also to prove various aspects and hypotheses of the concept [3-4].

\section{State-of-the-Art}

\subsection{Multi-modal folding bike commuting}

When it comes to multi-modal transport, cycling as a complement to public transport has various environmental, health, and traffic-reduction benefits [5-7], but it also has its challenges. For example, the relatively insecure parking at the station has its drawbacks [8]. It encourages the use of low-value, expendable bikes with often poor riding characteristics and a lack of safety and comfort [8]. In most cases, a commuter is required to have two bikes, one on both the departure location and one on the arrival location [6]. The perfect solution to these drawbacks is the use of folding bikes, enabling a commuter to retain the bicycle at all times as an item of personal luggage.

However, besides the clear benefits of folding bikes, the current market offer still has several downsides, such as the uncomfortable small wheel size [8], the oversized volume when folded and their excessive weight [9]. In literature, only very few studies were found concerning the user friendliness of folding bikes [9-10] or the user experience of folding bike using commuters. Nevertheless, the downsides mentioned above were demonstrated and reported by the multiple test subjects during the initial user tests.

\subsection{Folding bike design}

As regards the innovation and design of folding bikes, some literature studies can be found concerning folding bikes that have been commercialised, such as the design process of the Strida folding bike [11], concept studies that remained concepts, such as 'The Intermodal Bike' project [9] and more specific studies, such as the correct use of materials and production techniques for folding bikes [12].

An interesting study was conducted on the optimal direction of the pivot axis of the hinge of a folding bike [13]. An alternative was sought for the classic single lateral pivot type folding mechanism, which causes significant troubles in folding dimensions and the front and rear wheels end up arranged in a slanted way. The research shows for the need of a folding mechanism that aligns both the front and rear wheel in parallel, which would result in excellent usability and smaller folding width. A commonly occurring and remarkable phenomenon in patent research is the use of singlesided suspension, both for the front and rear wheel. In this way both wheels are positioned adjacently to each other with minimal width when the bike is folded [14-17].

\section{Method}

\subsection{Methodology}

Although inspiration was gained through several design methodologies, such as design thinking and user-centred design, an own method and structure was followed throughout the design process. First 
of all, the three aspects of design thinking (Figure 1), necessary for innovative design: business, technology and people were all constantly taken into account throughout the entire process [18].

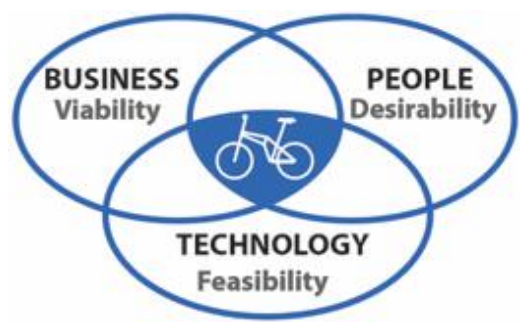

Figure 1. Three phases for innovative design.

The executed design process can be divided into three main phases. First there is the problem definition phase, in which market research and initial user tests are performed and the scope and research question of the thesis is defined. This is then followed by the developing phase that includes various iterative steps of ideation and prototyping. The final phase of the design process is the testing phase, in which several user tests were performed.

\subsection{Problem definition phase}

The problem definition was initiated with market research on the current offer of folding bikes, from which the market opportunity was derived (Figure 2).

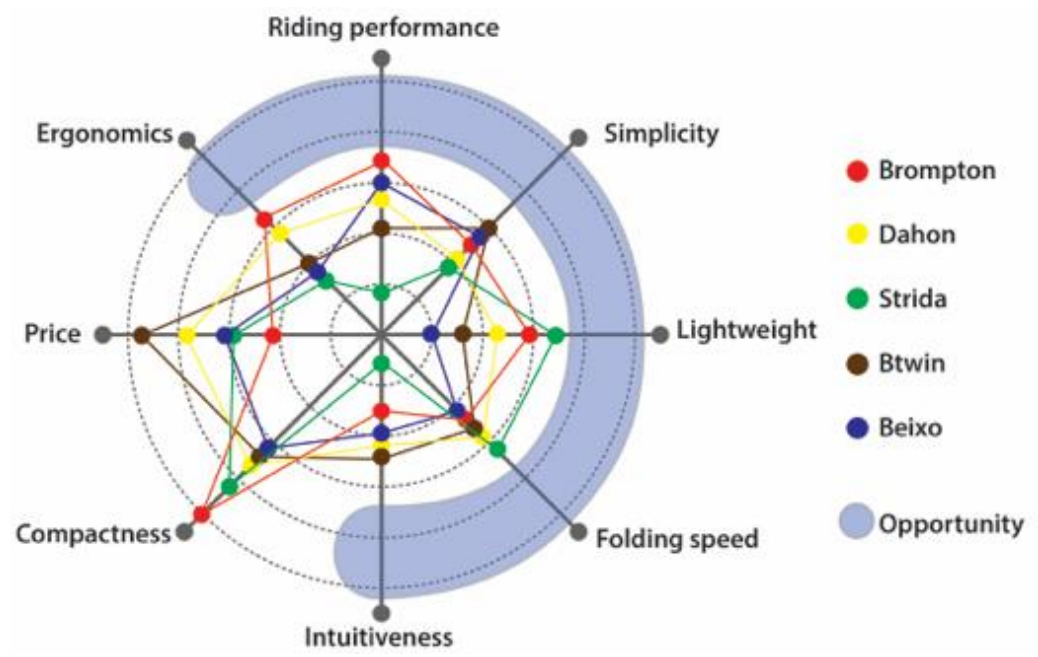

Figure 2. Three phases for innovative design.

Besides, a specific target group was chosen, namely young commuters, aged between 25 and 45 , since this is the target group that matches best with the found market potential. In order to achieve a folding bike that works best for these users, User Experience Design techniques were used [19-20]. Personas were made to summarise the findings and research about the stakeholders [21]. A customer journey was drafted to get a better understanding of the specific context in which folding bikes are used by commuters and the actions that are performed in each part of this customer journey (Figure 3 ) [22-23]. 


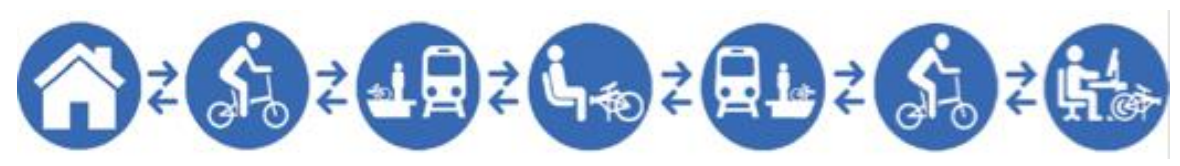

Figure 3. The customer journey of a folding bike using commuter.

Both the persona and customer journey were used as guidelines throughout the whole design process and the user tests. This contextual design lead to a better implementation of the specific demands and wishes of the stakeholders during each aspect of the customer journey [24].

All findings of the market research and the initial user tests were then summarized and converted to the focus of the concept bike to be designed.

\subsection{Developing phase}

The first ideation phase started by brain-sketching ideas and solutions for sub-problems found in the problem definition phase. From there several concepts were derived, with the focus on the overall shapes of the bikes and a corresponding rough idea of compacting/folding the bike. Through further sketching and the use of quick and dirty scale models, the abundance of ideas was narrowed down to three main concepts, of which one main concept was chosen in consultation with the stakeholders. After further developing, prototyping and optimising the folding mechanism, a first quick and dirty full-size prototype was made as a proof of concept. The goal of this prototype was to get a better way of testing and communicating the complete folding mechanism, dimensions and the usability with the stakeholders (Figure 4). The development of the full-size prototypes always started in CAD, in which first the standard components and the overall geometry were drawn and from there the folding bike's frame and fork were designed.
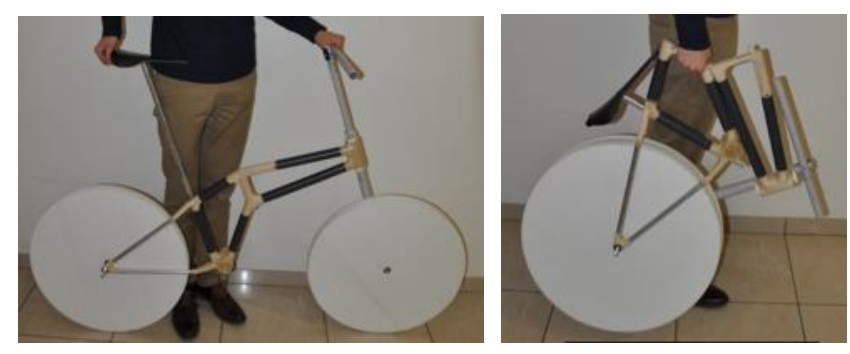

Figure 4. First quick and dirty full-size proof of concept.

Based on the learning outcomes of this first prototype, a second full-size prototype was made. The focus of this prototype was to optimise the dimensions and shape of the folding bike for structural rigidity, the incorporation of standard components and the overall ergonomics. Special attention was paid to make sure crucial bike geometry, such as the fork rake, the trail, the head tube angle and the bottom bracket height, are as optimal as possible in order to achieve the best bike handling characteristics. The goal of this prototype was to have a functioning bike, containing all the necessary standard components, enabling usability and user tests. Doing so, the strived improvement of the user experience of commuters and the usability can be researched. With the intent of fast, low budget easily adaptable prototyping, the frame and front fork were built out of carbon fibre tubes connected with $3 \mathrm{D}$ printed lugs.

\subsection{Testing phase}

Test offset wheels: One main aspect of the concept is that the front and rear wheels are both mounted with an opposing offset, so the two wheels are not perfectly aligned, but positioned in parallel to one another. This results in the two wheels coming right next to and against each other when folding the 
bike perfectly vertically, keeping the overall width minimal and enabling the folded bike to be dragged like a trolley on its two wheels. The influence of the opposing offset of the two wheels on the riding experience was tested through several pilot tests and a user test.

Pilot test 1: In the first test the offset between the front and rear wheel was obtained by mounting the front wheel of a bike next to the front fork, resulting in an offset of $90 \mathrm{~mm}$ (Figure 5).
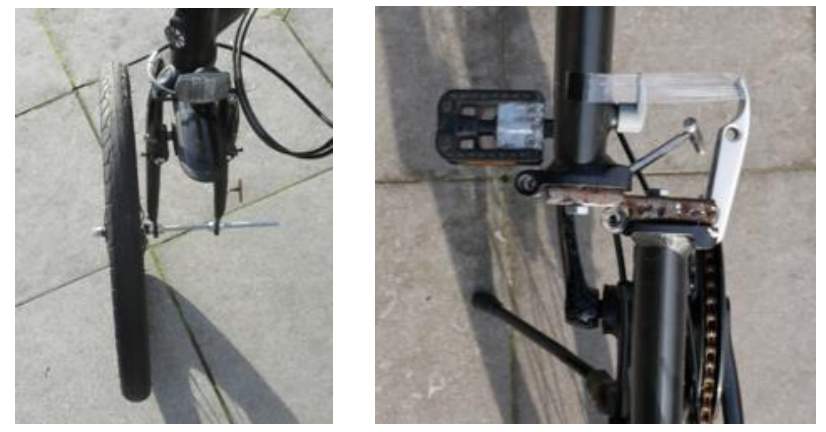

Figure 5. Pilot test 1 and 2 offset wheels

Pilot test 2: The goal of the second test was to create offset wheels, but with the head tube and thus the pivot point of the fork straight above the front wheel, as is normally the case in a bicycle. Now the parallel offset wheels are achieved by splitting a bike frame in half and mounting it with the desired $55 \mathrm{~mm}$ offset (Figure 5).

User test: After the successful initial findings of the second pilot test, a blind user test was conducted on 25 random test subjects without any foreknowledge of the design project (Figure 6). The user test was a comparative test between two very similar bikes, of which one bike was not adapted and one was adapted with parallel offset wheels. The frames of both bikes were identically wrapped so the offset, located in the frame of one of the bikes was not visually noticeable. The test subjects were asked to ride a given circuit with both bikes. Which bike they started with was randomly chosen. Afterwards a short interview was conducted with each participant on their findings about the riding experience with both bikes.
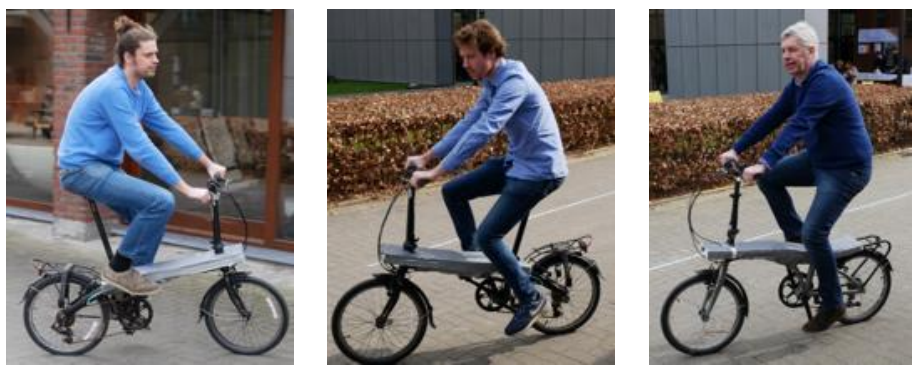

Figure 6. User test offset wheels.

One of the main aspects of improving the user experience of the commuters is to enhance the usability and ease of use of folding and unfolding the bike. This was tested with two new user tests.

Intuitiveness test: The first way this was analysed was by testing the intuitiveness of folding and unfolding the bike. This test is a comparative user test in which 20 test subjects, who had never seen the folding concept bike and had no association with it, have to fold and unfold three folding bikes for the first time, without any instructions or outside help (Figure 7). The three folding bikes consist of the concept folding bike and two commercial bikes (Brompton and Dahon) that represent the market offer. To get a broader view on the intuitiveness, half of the test subjects started with folded bikes and the other half started with unfolded bikes. The time it took to fold and unfold each bike was timed to be able to compare and analyse the test results. Besides that, the folding and unfolding of the concept 
folding bike was analysed during the test to gain insights in how users interpret the concept folding bike and to be able to improve intuitiveness and usability of the folding mechanism afterwards. A time limit of three minutes was determined through pilot tests.
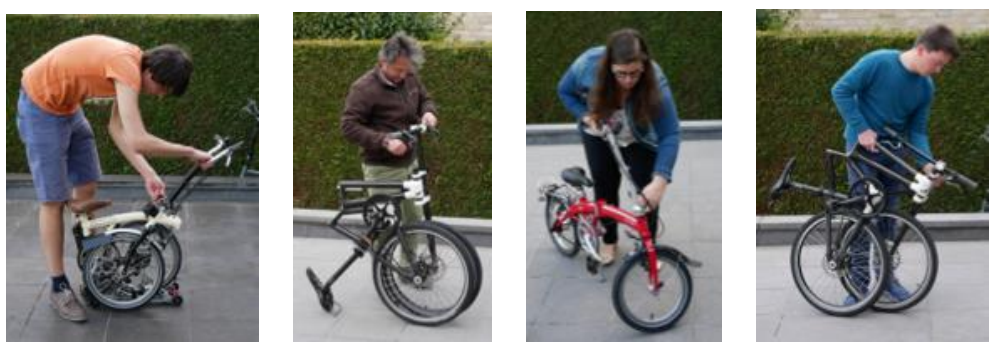

Figure 7. Intuitiveness and folding speed user tests.

Folding speed test: The second way to test the usability of folding and unfolding the bike was by performing a comparative test on the speed of folding and unfolding the bike. This test was executed by 20 test subjects after they had been demonstrated the correct way of folding each bike and once they got the hang of the folding motion (Figure 7). This test was also a comparative test between two commercially available folding bikes (Brompton and Dahon) and the concept folding bike. The folding and unfolding was timed to be able to compare and analyse the test.

Research was done to define which aspects determine the user experience of using a folding bike for commuting. The user experience and usability and all the different aspects combined, appeared to result in the overall complete user experience. This cannot be tested or judged through individual aspects alone; therefore, a fifth test was conducted.

Customer journey test: The fifth test is a user test focusing on the overall user experience of the stakeholder using the folding bike throughout the complete customer journey. In this test, three test subjects went through their complete commute, using the folding bike prototype (Figure 8). The full test was observed to analyse the test subjects' proceedings and the think-aloud protocol was applied to gain insights in the test subjects' thoughts [25]. Afterwards an interview was conducted to get a better understanding of participants' overall user experience of the folding bike during the commute.
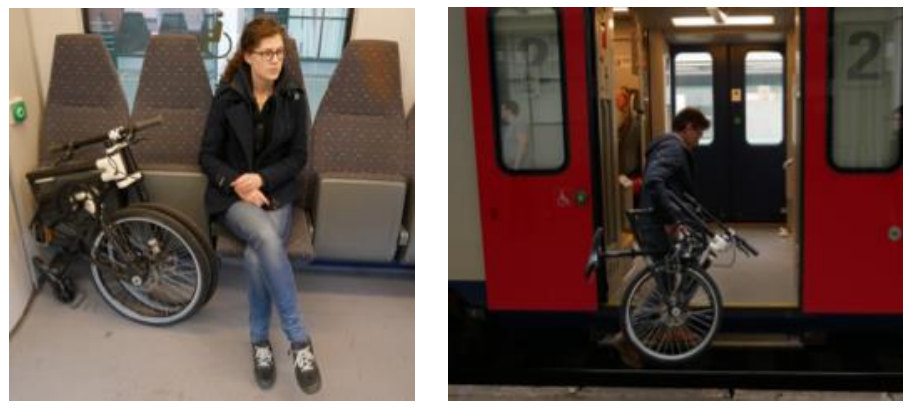

Figure 8. Usability test during customer journey commuter.

\section{Results}

\subsection{Developing results}

The result of the design project is a functional prototype with which the usability, the handling and the folding of the concept bike can be tested and the user tests can be executed (Figure 9). Despite the fact 
that cycling with the bike is not possible due to the production method used for this prototype, this aspect of the concept has been thoroughly tested in Test offset wheels.
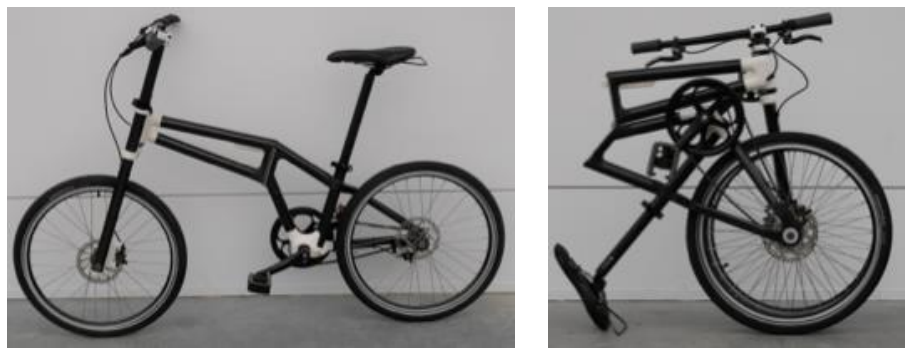

Figure 9. Final prototype of the concept folding bike.

A first main feature is the 24 inch ETRTO $507 \mathrm{~mm}$ sized wheels, which are an intermediate size between the traditional 20 -inch folding bike wheels and the classical 26 -inch city bike wheels. This results in the ultimate balance between comfort, stable handling characteristics on rough roads, high speeds and compactness after folding.

A second main feature is the simplicity and speed of frame folding. This is achieved by lifting the bike vertically on the provided handle. Thanks to this lifting motion, the lever is being squeezed and the locking mechanism unlocks. Once the locking mechanism is unlocked, the same upwards lifting motion causes the wheels of the bike to ride towards each other and the bike frame to fold without having to lift the complete weight of the bike (Figure 10). Unfolding the bike frame is operated by taking the handlebar and the seat post and by moving them towards each other. This results in the wheels of the bike riding away from each other and the bike locking mechanism closing and automatically locking. An additional advantage of this folding mechanism is the resilience. The weight of the bike and the weight of the rider push the locking mechanism closed. This way there is no need for an additional safety lock on the locking mechanism, resulting in a faster, more userfriendly and intuitive locking mechanism.
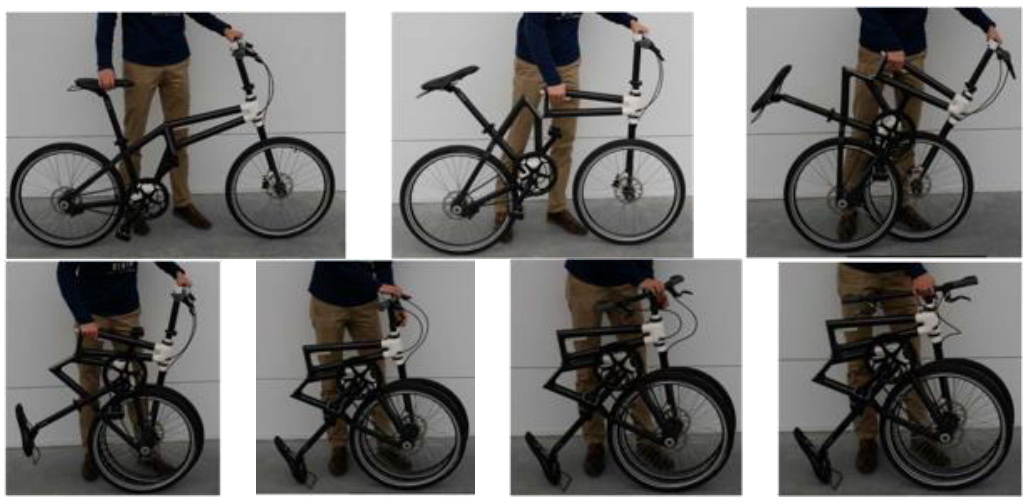

Figure 10. Final prototype of the concept folding bike.

A third main feature is the trolley function. After folding the bike frame and if desired also sliding in the seat post, the bike can be pulled like a trolley on the wheels, using the handlebar as a handle. That means the bike does not have to be carried when transported by foot.

A fourth main feature is the adjustability of the handlebar height that is integrated in the compacting mechanism of the handlebar and stem. This is done by sliding the telescopic stem into the front fork. In the first part of the movement, the stem slides straight into the fork. This makes the handlebar adjustable in height. In the second part of sliding in the stem, the handlebar makes a $90^{\circ}$ turn, causing the handlebar to end up against and parallel to the frame (Figure 10). This keeps the 
width of the folded bike as minimal as possible. A pin on the top of the front fork and the slot in the stem is used to guide the stem straight in the first section and then into a spiral curve.

\subsection{Testing results}

Pilot test 1 offset wheels: The first test shows that if the wheel is mounted on the right side of the fork, the bike has a tendency to turn left and vice versa. This can be explained by the bikes head tube, and thus the steering pivot point that is not straight above the front wheel. This results in the stable position not being straight forward, but a bike that constantly pulls to one side. Despite this, the test shows positive results regarding the riding experience, both riding straight and cornering.

Pilot test 2 offset wheels: This pilot test shows a perfectly stable bike, the offset of which does not impact the riding characteristics; it is even possible to ride no handed.

User test offset wheels: None of the 25 test subjects experienced a difference in riding experience or characteristics between the two test bikes caused by the $55 \mathrm{~mm}$ offset located in the frame of one of the test bikes. Furthermore, this offset was only noticed by 1 out of the 25 test subjects because of the offset being located in the middle of the frame, resulting in a slightly crooked riding position.

Intuitiveness test: The intuitiveness test shows an average folding speed of $105 \mathrm{~s}$ for the Brompton, 96s for the Dahon and 61s for the concept and an average unfolding speed of 116s for the Brompton, $72 \mathrm{~s}$ for the Dahon and 33s for the concept bike. During this intuitiveness test 3 out of the 20 test subjects succeeded in folding the Brompton correctly, 6 out of 20 folded the Dahon correctly and 19 out of 20 folded the concept bike correctly (Figure 11).

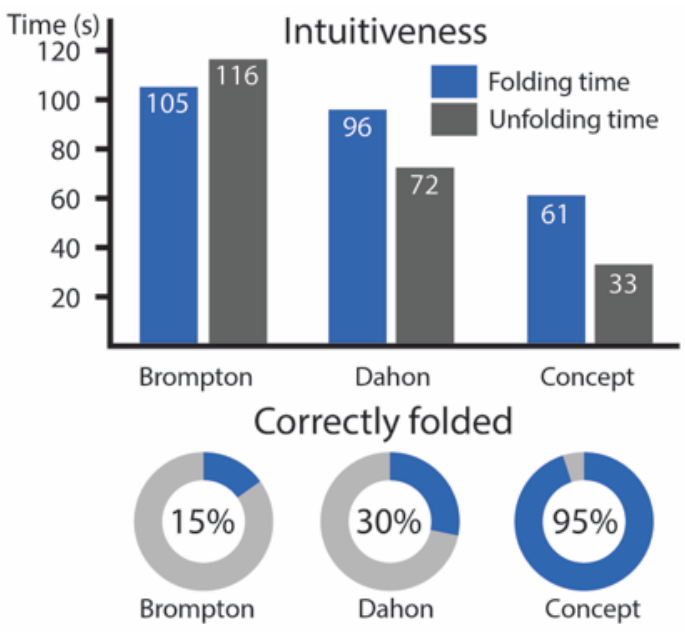

Figure 11. Test results user test intuitiveness.

Folding speed test: The folding speed test results in an average folding speed of $37 \mathrm{~s}$ for the Brompton, 37s for the Dahon and 17s for the concept bike and an average unfolding speed of 38s, 33s and 12s respectively (Figure 12). All test subjects experienced the concept bike to fold and unfold the quickest. 11 out of the 20 test subjects experienced the Dahon to fold and unfold second quickest and the Brompton the slowest, while the other 9 test subjects experienced the opposite. 


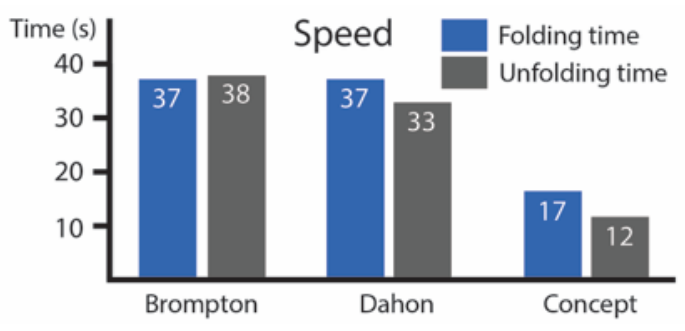

Figure 12. Test results user test folding speed.

Customer journey test: During the usability test in which the customer journey of a folding bike commuter was completed, some of the aspects allowing room for improvement were highlighted. For example, the magnet that keeps the wheels closed must be a lot stronger and custom wheels, of which the non-supported side is completely flat, are required for improved compactness and overall rigidity when folded. As expected, a telescopic seat post is required for smaller folding. Resting the bike on the seat instead of the extended handlebar when folded proved to be a more intuitive and better way to put down the bike when folded. Therefore, a bracket was designed and added to the seat. Moreover, the unique selling propositions of this bike, such as the fast and easy folding and the minimal weight, were valued by the test subjects as expected. Compared to the test results of the initial user test, an overall improvement of the user experience and the usability of using the folding bike was experienced by the stakeholders (Figure 13).

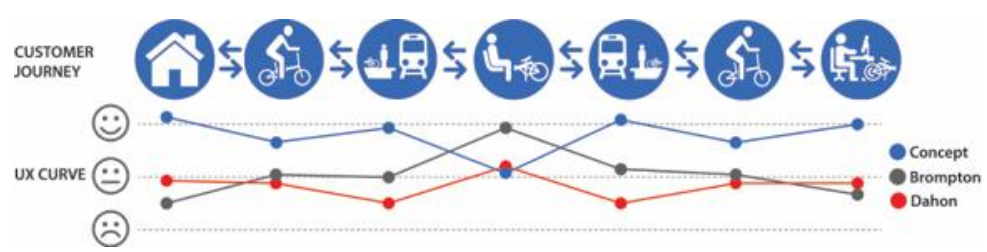

Figure 13. UX curve throughout the customer journey.

\section{Discussion}

User tests show that wheels aligned in parallel have no noticeable impact on the riding characteristics of a bike, provided the opposite offset mounted wheels are properly integrated in the bike's overall design. The offset was noticed by one test subject due to the crooked riding position caused by the offset being located in the frame. In the designed folding bike concept, the offset is located in the wheel mounting instead of in the bike frame and the riding position of the rider is thus straight and the offset unnoticeable.

The intuitiveness test shows that folding and especially unfolding the concept bike is a lot more intuitive than its two benchmarks. In addition, more participants succeeded in correctly folding the concept bike, which shows that it is more error-proof. The folding speed test shows that the concept bike is a lot quicker to fold and unfold than both benchmarks. All participants also experienced the concept bike to fold quicker. The participants however had limited practice time to master the folding movement. The folding speed of each bike can increase a little with more practice.

The results of both the intuitiveness test and the folding speed test indicate the folding and unfolding of the concept to be more user friendly and improve the user experience of folding the bike. The fragile prototype with 3D printed PLA lugs had a negative impact on the intuitiveness test, the folding speed test and the usability test since the participants were afraid of handling the prototype incorrectly and of damaging the frame. Apart from that, the prototype also presents room for improvement. Therefore, the executed user tests are no complete statistical tests, yet rather explorative tests performed in order to orient the design process. Further statistical tests require fully functional 
and properly constructed prototypes, of which the prototyping effort and costs should not be underestimated

\section{Conclusion and future work}

The design of an innovative folding bike concept can be seen as a big puzzle of standard components and self-designed components, in which certain traditional bicycle geometry aspects and rules have to be taken into account. For each standard component, an enormous amount of size and brand variations exist. A lot of foreknowledge is required to be able to select the right combination of standard components or universal dimensions. Not collaborating with a bicycle or folding bike company worked to the detriment of this aspect, but it enabled the design to be a lot more disruptive. During the design process, the consideration between custom wheels and standardised wheels was made. Due to time limitations of the thesis and the ease of use of standardised components, both for prototyping and in real life for bike repair, the preference was given to standardised wheels. The final prototype and design process however shows that a standard bicycle hub is still too wide for this application with offset wheels and single-sided suspension, resulting in the need for a very wide and thus custom bottom bracket and crank shaft. This, combined with the wide front fork and rear wheel mount, the overall folded prototype bike is a lot wider than initially intended. Therefore, custom bike rims with very narrow hubs and an inside mounted rear sprocket are a must for further development and improvement of this bike concept. Custom rims will also enable the inside of the bike wheel to be completely flat, so no hub protrudes. This will enable the two wheels to rest completely flat against each other without space for motion when the bike is folded.

A second suggestion for future work is researching and exploring the alternatives for production methods for the folding bike concept. Aspects such as the durability, weight and cost of production of different production techniques have to be compared. Within the field of composite manufacturing, different techniques such as $\mathrm{SMC} / \mathrm{BMC}$ and traditional prepreg monocoque lay-ups may be considered and combined. Also, the weigh-up has to be done between composites or other bike building materials such as aluminium, titanium or steel. The material choice also influences the folding bike design, which will have to be fine-tuned according to the chosen material and production technique. There are two main suggestions for material choice and production technique to produce the concept bike's frame.

The first possibility is a traditional monocoque frame, such as present-day racing bikes. To apply this production technique to the bike concept, the monocoque has to be divided in a front part and a rear part, isolated by the central locking mechanism. This central hinge and locking mechanism could be an aluminium milled or forged insert of a composite BMC insert in the monocoque frame. Moulding this monocoque frame will be easier than traditional bike frames due to the single sided rear wheel mount. This means only two mould halves are required for the rear part of the bike frame.

A second and cheaper variant to produce the bike frame, involving less manual labour and cheaper material costs, is a welded aluminium bike frame. The individual parts could be a combination of hydroformed panels with $\mathrm{CNC}$-milled or forged aluminium inserts for the central locking mechanism. The main selection criterion will be the production cost versus the weight of the frame.

There are also several potential materials to produce the custom bike rims, such as magnesium alloys, carbon fibre rims or other fibre reinforced plastics. In all variants, the bike rim would be bolted to a CNC-lathed wheel hub.

In this design process, the focus was on the user aspect of the folding bike design. When further developing this bike, attention will have to be paid to the safety standards and regulations. This will necessitate additional tests, such as the standardised fatigue tests, strength tests, corrosion tests etc. These mechanical tests require fully functional and correctly constructed prototypes. This entails high prototyping effort, costs and foreknowledge. To conclude, for these next steps in the development process, a collaboration with an established bicycle manufacturer would be highly recommended. 


\section{References}

1. The Folding Cyclist, "Folding Bike News," (2017). Available: http://www.foldingcyclist.com/. [Accessed: 03-May-2017].

2. L. Cooke, J. Roderiguez, M. Chino, L. Wang, J. Fehrenbacher, C. Cameron, J. Dloffa, K. Lofgren, L. Cowan, P. Rebecca, L. Evelyn, A. Schwartz, M. Chino, and J. Chapa, "Folding Bike | Inhabitat - Green Design, Innovation, Architecture, Green Building." Available: http://inhabitat.com/tag/folding-bike/. [Accessed: 03-May-2017].

3. M. F. Story, "Maximizing Usability: The Principles of Universal Design," Assist. Technology, 10, 1, pp. 4-12, (1998).

4. [4] D. J. Mayhew, "The Usability Engineering Lifecycle," in CHI '99 Extended Abstracts on Human Factors in Computing Systems, pp. 147-148. (1999)

5. K. J. Krizek and E. W. Stonebraker, “A Marriage Unrealized”, pp. 161-167, (2010).

6. P. Rietveld, "Non-motorised modes in transport systems: a multimodal chain perspective for The Netherlands," Transp. Res. Part D Transp. Environ., vol. 5, no. 1, pp. 31-36, (2000).

7. Y.-H. Cheng and K.-C. Liu, "Evaluating bicycle-transit users' perceptions of intermodal inconvenience," Transp. Res. Part A Policy Pract., vol. 46, no. 10, pp. 1690-1706, (2012).

8. P. Cox, "The Role of Human Powered Vehicles in Sustainable Mobility," vol. 34, no. 2, (2005).

9. F. Tosi, A. Belli, A. Rinaldi, and G. Tucci, "The Intermodal Bike : multi-modal integration of cycling mobility through product and process innovations in bicycle design," vol. 41, pp. 15011506, (2012).

10. M. Arunachalam, "A Typical Approach in Conceptual and Embodiment Design of Foldable Bicycle," vol. 87, no. 19, pp. 9-16, (2014).

11. R. Roy, "Case studies of creativity in innovative product development," Des. Stud., vol. 14, no. 4, pp. 423-443, (1993).

12. M. A. Maleque, M. S. Hossain, and S. Dyuti, "Material Properties and Design Aspects of Folding Bicycle Frame," vol. 265, pp. 777-782, (2011).

13. [13]J. Hyeong, J. Roh, and S. Kim, "Optimization of pivot obliquity for a foldable bicycle," Int. J. Precis. Eng. Manuf., vol. 17, no. 7, pp. 931-936, 2016.

14. [14] M. C. Chen, "Bicycle foldable to align front and rear wheels along a transverse direction of the bicycle." Google Patents, (2005).

15. P. P. M. \}ik, "Universal folding bicycle." Google Patents, (2004).

16. M. A. Jayasuriya, J. C. Cheng, and K. M. Schondorf, "Klapprad Folding bike." Google Patents, (2015).

17. D. K. De Jong, "Foldable bicycle." Google Patents, (2001).

18. T. W. Brown Jocelyn, "Design Thinking for Social Innovation," Dev. Outreach, vol. 12, no. 1, pp. 29-43, (2010).

19. D. Norman, "Emotion \& Design: Attractive Things Work Better," interactions, vol. 9, no. 4, pp. 36-42, (2002).

20. M. Maguire, "Methods to support human-centred design," Int. J. Hum. Comput. Stud., vol. 55, no. 4, pp. 587-634, (2001).

21. J. E. Nieters, S. Ivaturi, and I. Ahmed, "Making Personas Memorable," in CHI '07 Extended Abstracts on Human Factors in Computing Systems, pp. 1817-1824, (2007).

22. S. Nenonen, H. Rasila, and J. Junnonen, "Customer Journey - a method to investigate user experience," no. Schmitt (1999).

23. "Experience-Maps-Using-Customer-Journey-Maps-to-Improve-Customer-Experience.pdf."

24. H. Beyer and K. Holtzblatt, "Contextual Design," interactions, vol. 6, no. 1, pp. 32-42, (1999).

25. J. Nielsen, T. Clemmensen, and C. Yssing, "Getting Access to What Goes on in People's Heads?: Reflections on the Think-aloud Technique," in Proceedings of the Second Nordic Conference on Human-computer Interaction, pp. 101-110, (2002. 